\title{
Compensation of Irregular Currents with Active Filters
}

\author{
Leon M. Tolbert, Senior Member, IEEE, Yan Xu, Student Member, IEEE, Jianqing Chen, Student Member, IEEE \\ Fang Z. Peng, Senior Member, IEEE, and John N. Chiasson, Member, IEEE 1
}

\begin{abstract}
Compensation of irregular currents such as those associated with arc furnaces, transient disturbances, and power electronic converters is presented. The compensation is based on a well-defined non-active current definition. The basic concepts required in the definition of non-active current are presented and illustrated by compensation simulations for a variety of different types of non-periodic currents found in distribution electrical systems, including disturbance, subharmonic, and stochastic currents. Further, based on the compensation objectives for different types of load waveforms, the specifications such as current ratings or capacitance requirements of the active filter are also presented.
\end{abstract}

Index Terms - active filter, non-periodic current, arc furnace, non-active power definition.

\section{INTRODUCTION}

$\mathrm{T}$ HE widespread use of non-linear loads has increased the non-sinusoidal and non-periodic currents/voltages in power systems. Arc furnaces[6], transient disturbances, and power electronics converters such as cycloconverters and line-commutated three-phase thyristor-based rectifiers are the most common non-linear loads. Their line currents may contain both sub-harmonics (frequency lower than the line frequency) and super-harmonics (frequency higher than the line frequency but not the integer multiple). These currents are considered as non-periodic, though mathematically they may still have a periodic waveform. The period of the currents is not equal to that of the line voltage [8], [9].

Most previous efforts [1]-[5] focused on the compensation of periodic non-sinusoidal currents. The diversified features of non-periodic currents make the compensation quite difficult, and theoretically, the compensation is different than that for periodic currents. However, practically the two cases are very similar [7], and in both cases a sine wave with a constant rms magnitude is preferred for the source current after compensation.

If using a shunt active filter as the compensator, the compensator injects currents that are the difference between the desired source currents and the required load currents. Starting from this viewpoint, the work here characterizes the required compensation current, and in particular, determines

L. M. Tolbert, Y. Xu, J. Chen, and J. N. Chiasson are with the Department of Electrical and Computer Engineering, The University of Tennessee, Knoxville, TN 37996-2100 USA (e-mail: tolbert@utk.edu, yxu3@utk.edu, jchen5@utk.edu, chiasson@utk.edu).

F. Z. Peng is with the Department of Electrical and Computer Engineering, Michigan State University, East Lansing, MI 48824-1226 USA (e-mail: fzpeng@msu.edu). the energy storage requirements for various non-periodic currents based on a new definition of non-active current [5].

\section{DEFINITION OF NoN-ACTIVE CURRENT}

For a single or polyphase system, a shunt compensator to minimize the non-active power/current required of the source can be configured as in Fig. 1. It is assumed here that the compensator consists only of passive components (inductor and/or capacitor) and/or switching devices and no external power source. Consequently, conservation of energy requires the active power of the compensator to average zero (neglecting the compensator's power loss). In more detail, let $p_{S}(t), p_{L}(t)$, and $p_{C}(t)$ denote the instantaneous power of the source, load and compensator, respectively and their average values over a time interval $T_{C}$ be given by

$$
P_{X}(t)=\frac{1}{T_{C}} \int_{t-T_{C}}^{t} p_{X}(\tau) d \tau \text { where } X=S, L, \text { or } C .
$$

then

$$
P_{S}(t)+P_{C}(t)=P_{L}(t)
$$

and

$$
P_{S}(t)=P_{L}(t), P_{C}(t)=0 \text { as } \mathrm{t} \rightarrow \infty .
$$

In (1), the averaging interval $T_{C}$ varies depending on the compensation objectives and capacitance rate of the compensator. The subscripts " $S$ ", " $L$ ", and " $C$ " denote the source, load, and compensator quantities as shown in Fig. 1, respectively. Based on these physical and practical limitations, non-active power/current can be defined and formulated.

Fryze's idea of non-active current/power [4] is extended:

$$
i_{p}(t)=\frac{P_{L}(t)}{V_{P}^{2}(t)} v_{P}(t), \quad i_{q}(t)=i(t)-i_{p}(t),
$$

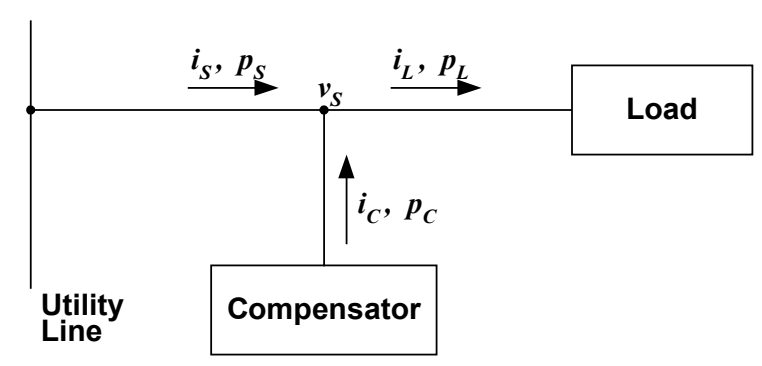

Fig. 1. A shunt compensator for current compensation. 
and

$$
V_{P}(t)=\sqrt{\frac{1}{T_{C}} \int_{t-T_{C}}^{t} v_{P}^{2}(\tau) d \tau} .
$$

where $i_{p}(t)$ is the active current, $i_{q}(t)$ is the reactive or nonactive current. $P_{L}(t)$ is the average active power over the interval $\left[t-T_{C}, t\right]$, which is calculated from $(1) . V_{P}(t)$ is the rms value of the voltage calculated using $v_{P}(t)$ over the interval $\left[t-T_{C}, t\right]$ as in (5). Here $v_{P}(t)$ is the reference voltage whose specification depends on the compensation objectives. For example, this specification can be the utility voltage $v_{s}(t)$ itself, or it may be the fundamental component of $v_{s}(t)$ (i.e., $v_{P}(t)=v_{f}(t)$, where $v_{s}(t)=v_{f}(t)+v_{h}(t), \quad v_{f}(t)$ is the fundamental, and $v_{h}(t)$ is the harmonic component). The definitions (4) and (5) are valid for single- and polyphase circuits. However, in the case of polyphase circuits, the voltages and currents are expressed in vector form which, for a three-phase system, are

$$
v=\left[v_{a}, v_{b}, v_{c}\right]^{T}, \quad i=\left[i_{a}, i_{b}, i_{c}\right]^{T}
$$

and $\quad v^{2}=\left[v_{a}, v_{b}, v_{c}\right] \cdot\left[v_{a}, v_{b}, v_{c}\right]^{T}=\left(v_{a}{ }^{2}+v_{b}{ }^{2}+v_{c}{ }^{2}\right)$.

Table I shows that by choosing different voltage reference and $T_{C}$, different source currents will result. Because of its flexibility in regards to compensation objectives, this definition is quite suitable for analyzing the compensation of non-periodic currents found in many electrical distribution systems. This will be shown in the next section.

TABLE I. PARAMETERS FOR DIFFERENT COMPENSATION OBJECTIVES

\begin{tabular}{|l|c|c|l|}
\hline $\begin{array}{l}\text { Compensation } \\
\text { Objective }\end{array}$ & $\boldsymbol{v}_{\boldsymbol{P}}$ & $\boldsymbol{T}_{\boldsymbol{C}}$ & Resulting Source Current \\
\hline $\begin{array}{l}\text { Single phase reactive } \\
\text { current }\end{array}$ & $v$ & $T / 2$ or $T$ & $\begin{array}{l}\text { Unity pf and sinusoidal for } \\
\text { sinusoidal } v_{s}\end{array}$ \\
\hline $\begin{array}{l}\text { Single phase reactive } \\
\text { power and harmonic } \\
\text { current }\end{array}$ & $v_{f}$ & $T / 2$ or $T$ & $\begin{array}{l}\text { Unity pf and sinusoidal } \\
\text { regardless of } v_{s} \text { distortion }\end{array}$ \\
\hline $\begin{array}{l}\text { Instantaneous reactive } \\
\text { power for polyphase } \\
\text { system }\end{array}$ & $v$ & $T_{C} \rightarrow 0$ & $\begin{array}{l}\text { Instantaneously unity pf } \\
\text { for polyphase system }\end{array}$ \\
\hline $\begin{array}{l}\text { Non-periodic and } \\
\text { disturbance current }\end{array}$ & $v_{f}$ & $n T$ & $\begin{array}{l}\text { Smoothed sine wave with } \\
\text { unity pf }\end{array}$ \\
\hline
\end{tabular}

\section{IRREgUlaR CURRENT COMPENSATION}

For compensation of periodic currents with fundamental period $T$, choosing different $T_{C}$ alone does not change the source current characteristics. With reference to (5), the rms value of a periodic quantity does not depend on the time averaging interval $T_{C}$ if it is an integer multiple of $T / 2$.

Theoretically, $T_{C}$ can be chosen as any arbitrary value in the case of non-periodic currents. However, it is desirable for the interval to be an integer multiple of the line frequency period because of the desire that the source current be sinusoidal and have the same frequency as the source voltage frequency. In general, the period of the line voltage is not the same as the period of the quasi-periodic current $i_{p}(t)$. Thus, different $T_{C}$ will result in quite different source and compensator currents. Simulation results of the compensation of three kinds of non-periodic currents are given in the following subsections.

\section{A. Non-periodic Disturbance Currents}

The duration of a non-periodic current may be a fraction or multiples of the line frequency cycle. For the remaining period, the current may be zero or sinusoidal. Figs. 2, 3 show the simulation result of a single-phase disturbance current when $T_{C}=T / 2$ (Fig. 2) and $T_{C}=2 T$ (Fig. 3), and $v_{P}=v_{f}$ for both cases. To simplify the simulation, the voltage is assumed to be a sine wave. However, the result will also apply in a non-sinusoidal source voltage with harmonics.

Since the disturbance energy is fixed, different $T_{C}$ results in different average active power $P_{L}$; and thus different source and compensator current. Larger $T_{C}$ results in smaller peak values of $\left|i_{s}\right|$, i.e., smaller disturbance (or demand) seen from the source, but the compensator current rating will increase as seen in Fig. 6. The tradeoff must be weighed between minimizing the source current against additional capacitance. While the simulations are for single-phase, the same result would be expected in three-phase cases.

\section{B. Quasi-periodic (or Sub-harmonic) Currents}

One important feature of these non-periodic currents is that the current may have a repetitive period. In Fig. 4, the quasiperiodic current is composed by adding a sub-harmonic $(10 \mathrm{~Hz})$ to the fundamental current $(60 \mathrm{~Hz})$. The simulation shows that when $T_{C}$ is longer than or equal to a multiple of $1 / 2$ of the common period of the sub-harmonic and the fundamental $(20 \mathrm{~Hz}$ in this example), the source current is sinusoidal (Fig. 4(c)). If $T_{C}$ is shorter than that value, the source current still contains sub-harmonic components; as a result, the currents will not have the same amplitude cycle to cycle as can be seen in Fig. 4(b).

\section{Stochastic Non-Periodic Currents}

The currents of arc furnaces are irregular (Fig. 5), no finite $T_{C}$ exists to get a sinusoidal source current. Mathematically, the current period is infinite. From (3), (4), if $T_{C}$ is infinity, $P_{L}$ and $\mathrm{V}_{p}$ become constant and $i_{p}$ tracks $v_{p}$. If $v_{p}$ is chosen as $v_{f}, i_{p}$ will be sinusoidal. While an infinite $T_{C}$ is infeasible in practice, it is still possible to find some repetitive period in the current that has most of the rms content. Choosing a long $T_{C}$ may get an acceptable source current which is quite close to a sine wave as shown in Fig. 5 where $T_{C}$ is chosen to be 10 times the voltage fundamental frequency for near-complete compensation. Thus, the voltage flicker and harmonic penetration problem $[10,11]$ associated with this nonperiodic current can be mitigated. 


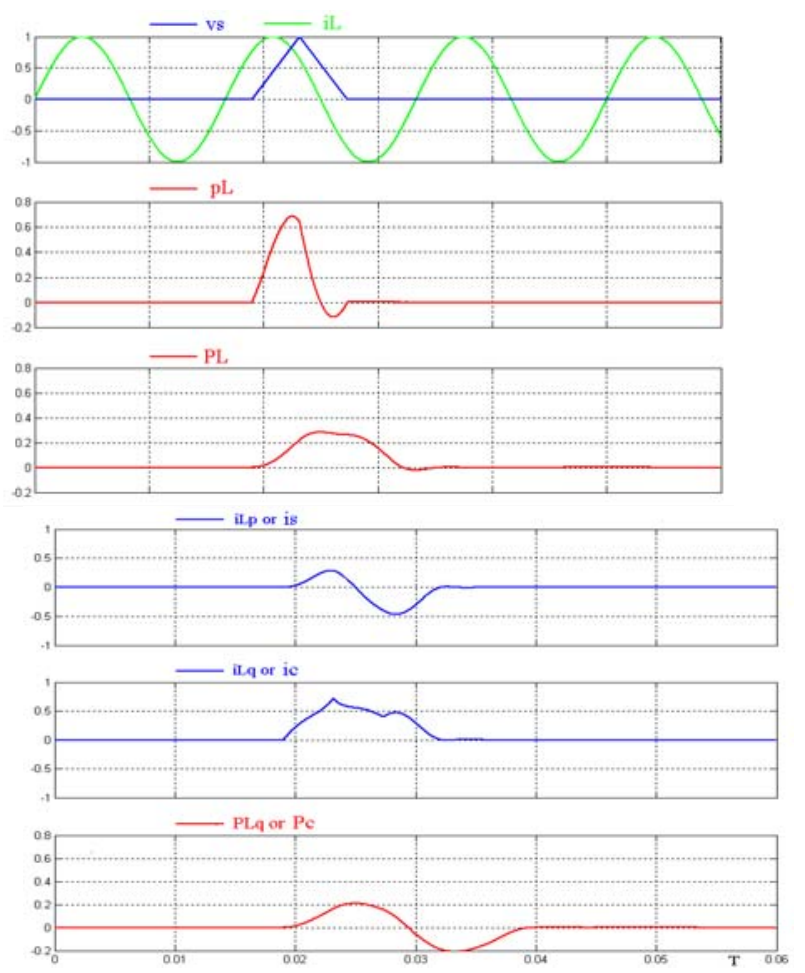

Fig. 2. Simulation results for disturbance type non-periodic current compensation $(T c=T / 2)$.

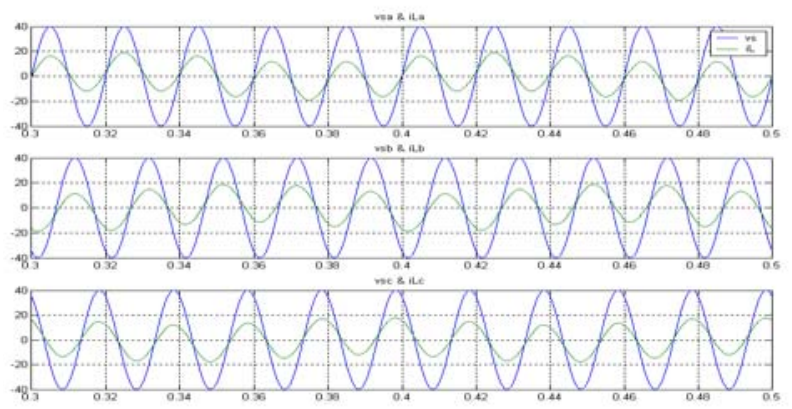

(a) 3-phase load current and voltage waveforms.

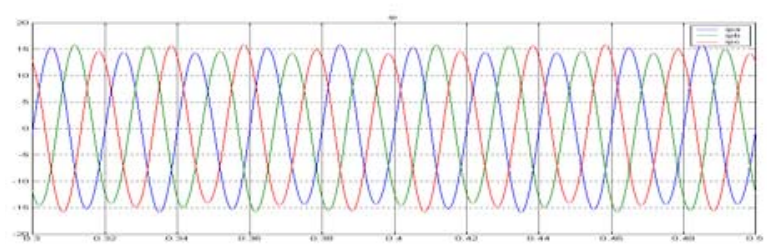

(b) Compensated load currents $(T c=T)$

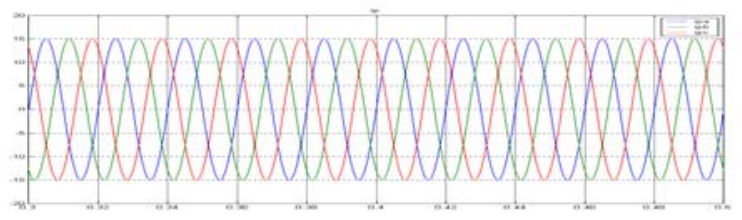

(c) Compensated load current $(T c=3 T)$

Fig. 4. Simulation of subharmonic current compensation.
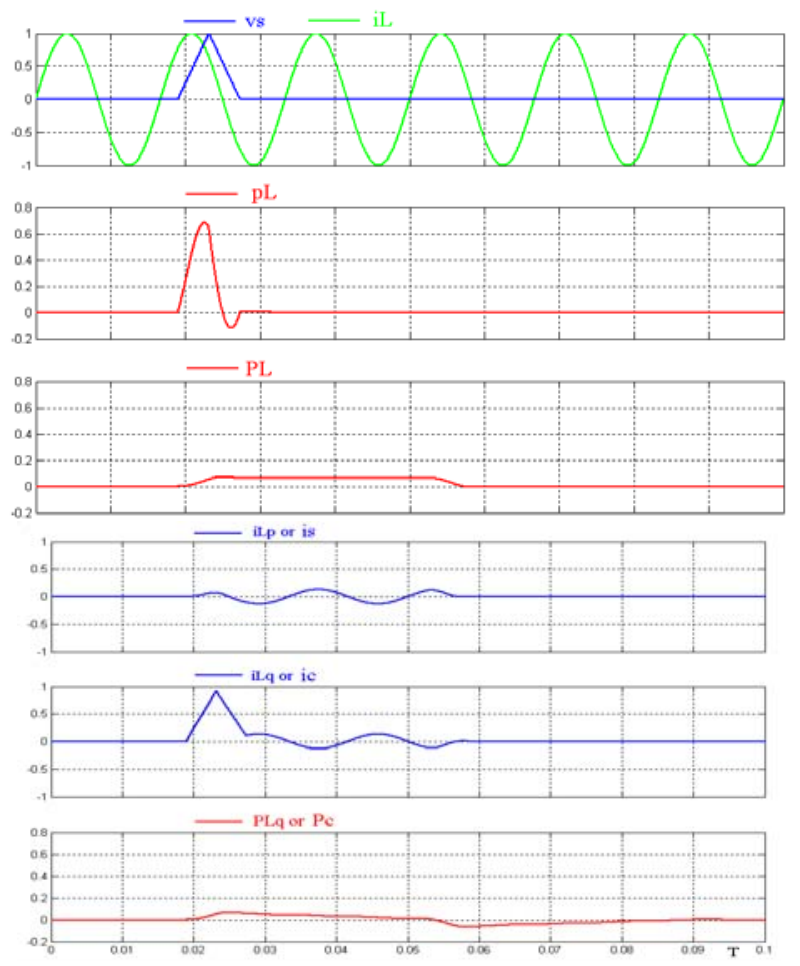

Fig. 3. Simulation results for disturbance type non-periodic current compensation $(T c=2 T)$.

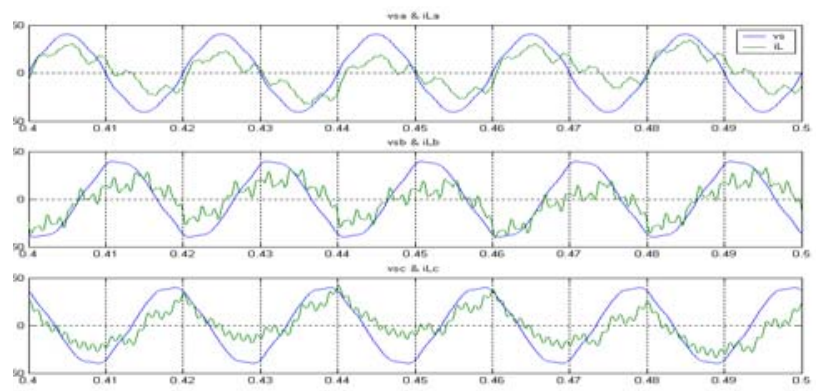

(a) 3-phase load current and voltage waveforms.

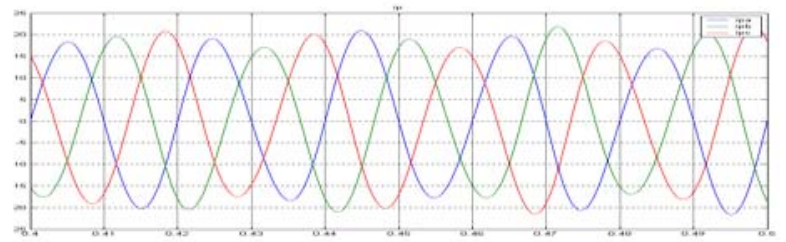

(b) Compensated load currents $\left(T_{C}=T\right)$

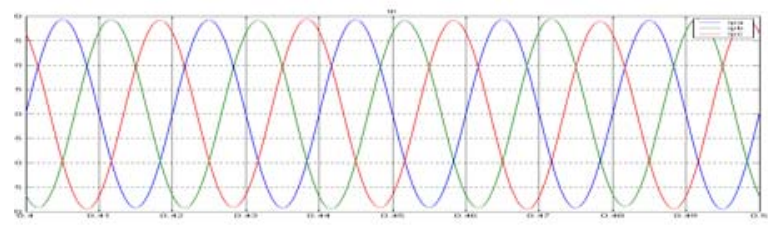

(c) Compensated load current $(T c=10 T)$

Fig. 5. Simulation of stochastic current compensation. 


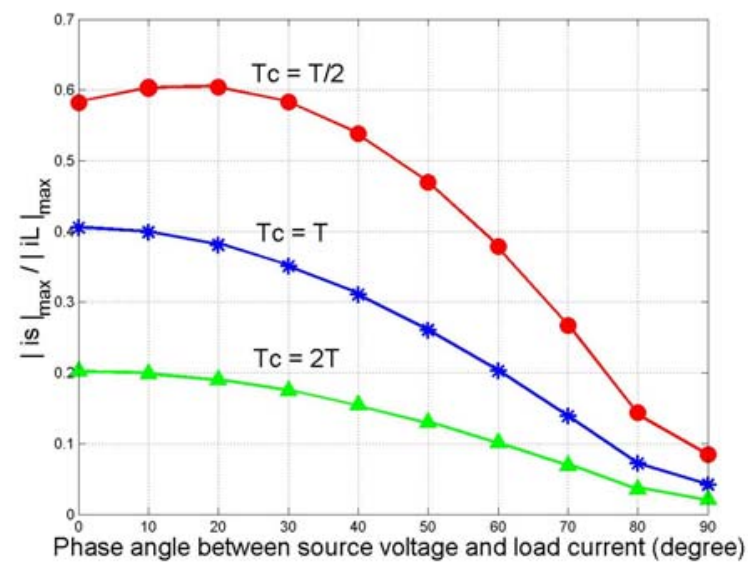

(a)

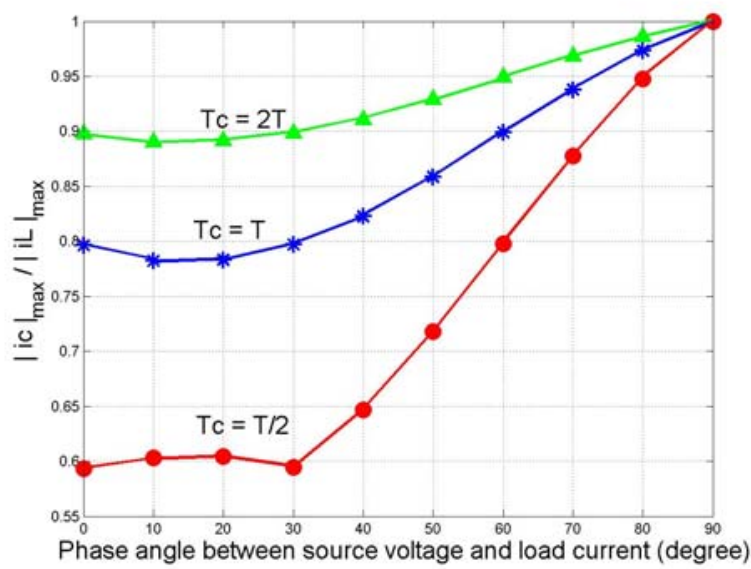

(b)

Fig. 6. (a) Peak source current and (b) peak compensator current normalized with respect to load current for different compensation times and load current phase angles.

\section{COMPENSATOR ENERGY STORAGE REQUIREMENTS}

According to the definition in (3), the average power of the compensator $P_{C}(t)$ over $T_{C}$ is zero.

$$
P_{C}(t)=\frac{1}{T_{C}} \int_{t-T c}^{t} v^{T}(\tau) i_{c} d \tau=0
$$

It has been shown in [5] that $P_{C}(t)$ is exactly zero in the periodic current case. However, during the interval $T_{C}$, the instantaneous power is not necessarily zero. The compensator generally has a capacitor for energy storage, and this capacitor has two processes: charge and discharge. Different capacitance is required to fulfill different compensation tasks. The maximum energy stored in the capacitor is at $t_{\max }$, when the capacitor changes from charge to discharge:

$$
\Delta E=\int_{0}^{t_{\max }} v^{T} i_{q} d t
$$

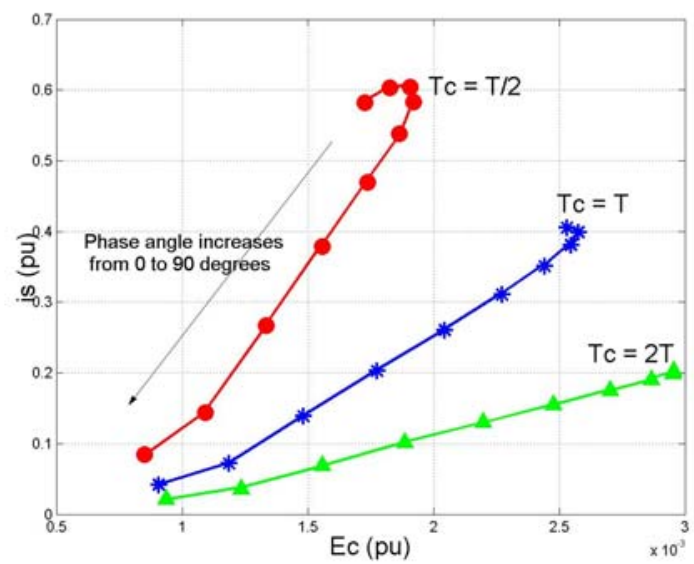

(a)

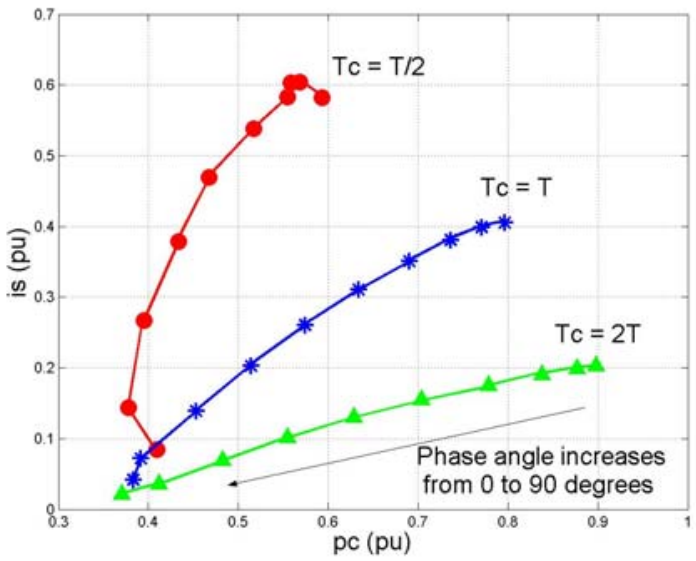

(b)

Fig. 7. Peak source current plotted as a function of the compensator's (a) energy storage requirement and (b) instantaneous power requirement for different compensation times and load current phase angles.

The capacitance required of the static var compensator can then be found as a function of the stored energy and voltage rating as $\mathrm{C}=2 \Delta \mathrm{E} / \mathrm{V}^{2}$. The load active power varies for nonperiodic currents.

If a sinusoidal current is desired, the instantaneous power difference between the load and source has to be supplied by the compensator, and a large capacitor is required. The choice of $T_{C}$ is also significant for the design of the capacitor. A longer $T_{C}$ results in a smoother source current with smaller amplitude; this requires that the compensator current $i_{c}$ increase as well as the capacitance requirement.

Fig. 6 shows the peak source current normalized with respect to load current for various compensation periods $T_{C}$ and phase angles between the pulse of current and source voltage for an example like that illustrated in Figs. 2 and 3. With $T_{C}$ changing from $T / 2$ to $2 T, i_{s}$ decreases from 0.6 to 0.2 p.u., while $i_{c}$ increases only from 0.6 to 0.9 p.u.

When the phase angle between the source voltage and current is small, which is common for many systems, a small increase in capacitance results in a much better compensation 
(i.e., much smaller source current). In the case of a large phase angle with $T_{C}=2 T, i_{c}$ increases $10 \%$ ( 0.9 to 1.0 p.u.); while for $T_{C}=T / 2, i_{c}$ increases $70 \%$ ( 0.6 to 1.0 p.u.).

Fig. 7 shows the peak source current plotted as a function of the compensator's energy storage requirement and instantaneous power requirement for various compensation times and load current phase angles. At longer $T_{C}$, the power drawn from the source is distributed during $T_{C}$ so that it has a smaller peak value instead of a short duration, high pulse. The compensator instantaneous power is more concentrated so that a larger capacitor is needed when the compensation duration is short. Over the complete compensation period $T_{c}$, the compensator provides only reactive power and does not consume or generate any active power because the load energy is always provided by the source.

\section{CONCLuSIONS}

Compensation of non-periodic currents is feasible using conventional shunt active power filters that are controlled based on a definition of non-active currents as described in this paper. Simulation results for compensation of various kinds of non-periodic currents gives credibility to the applicability of the definition for active compensators at many different types of loads. The choice of different averaging time intervals for the compensator determines the energy storage requirements and the extent of residual distortion in the source current.

\section{ACKNOWLEDGEMENT}

We would like to thank the National Science Foundation for partially supporting this work through contract NSF ECS0093884.

\section{REFERENCES}

[1] H. Akagi, Y. Kanazawa, A. Nabae, "Instantaneous Reactive Power Compensators Comprising Switching Devices without Energy Storage Components," IEEE Trans. Ind. Appl., vol. 20, May/June 1984, pp. 625-630.

[2] L. Rossetto, P. Tenti, "Evaluation of Instantaneous Power Terms in Multi-Phase Systems: Techniques and Application to Power Conditioning Equipment," ETEP, vol. 4, no. 6, Nov./Dec. 1994.

[3] F. Z. Peng, J. S. Lai, "Reactive Power and Harmonic Compensation Based on the Generalized Instantaneous Reactive Power Theory for Three-Phase Power Systems," Proceedings of the 7th International Conference on Harmonics and Quality of Power, Las Vegas, NV, October 16-18, 1996, pp. 83-89.

[4] L. M. Tolbert, T. G. Habetler, "Survey of Active and Non-Active Power Definition," IEEE International Power Electronics Congress, October 15-19, 2000, Acapulco, Mexico, pp. 73-79.

[5] F. Z. Peng, L. M. Tolbert, Z. Qian, "Definitions and Compensation of Non-Active Current in Power Systems," IEEE Power Electronics Specialists Conference, Cairns, Australia, June 23-27, 2002, pp. 17791784.

[6] G.Carpinelli, M.Di Manno, al., "AC and DC Arc Furnaces: A Comparison on Some Power Quality Aspects," IEEE Power Engineering Society Summer Meeting, 1999, pp. 499-506.

[7] H.Akagi, "Active filters and Energy Storage Systems Operated under
Non-periodic Conditions," IEEE Power Engineering Society Summer Meeting, Seattle, Washington, July 15-20, 2000, pp. 965-970.

[8] E.H. Watanabe, M.Aredes, "Compensation of Non-Periodic Currents Using the Instantaneous Power Theory," IEEE Power Engineering Society Summer Meeting, Seattle, Washington, July 15-20, 2000, pp. 994-999.

[9] L. S. Czarnecki, "Non-Periodic Currents: Their Properties, Identification and Compensation Fundamentals," IEEE Power Engineering Society Summer Meeting, Seattle, Washington, July 15-20, 2000, pp. 971-976.

[10] S. R. Mendis, M. T. Bishop, J. F. Witte, "Investigations of Voltage Flicker in Electric Arc Furnace Power Systems," IEEE Industry Applications Magazine, vol. 2, no. 1, Jan./Feb. 1996, pp. 28-34.

[11] M. M. Morcos, J. C. Gomez, Flicker sources and mitigation, IEEE Power Engineering Review, vol. 22, no. 11, Nov. 2002, pp. 5-10.

\section{BIOGRAPHIES}

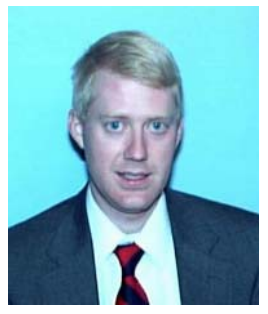

Leon M. Tolbert (S 1989 - M 1991 - SM 1998) received the B.E.E., M.S., and Ph.D. in Electrical Engineering from the Georgia Institute of Technology, Atlanta, Georgia

He joined the Engineering Division of Lockheed Martin Energy Systems in 1991 and worked on several electrical distribution projects at the three U.S. Department of Energy plants in Oak Ridge, TN. In 1997, he became a research engineer in the Power Electronics and Electric Machinery Research Center at the Oak Ridge National Laboratory. In 1999, he was appointed as an assistant professor in the Department of Electrical and Computer Engineering at the University of Tennessee, Knoxville. He is an adjunct participant at the Oak Ridge National Laboratory and conducts joint research at the National Transportation Research Center (NTRC). He does research in the areas of electric power conversion for distributed energy sources, motor drives, multilevel converters, hybrid electric vehicles, and application of $\mathrm{SiC}$ power electronics.

Dr. Tolbert is a registered Professional Engineer in the state of Tennessee. $\mathrm{He}$ is the recipient of a National Science Foundation CAREER Award and the 2001 IEEE Industry Applications Society Outstanding Young Member Award. He is an associate editor of the IEEE Power Electronics Letters.

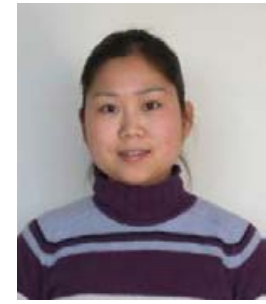

Yan Xu (S 2002) received the B.E. in Electric Power Engineering from Shanghai Jiaotong University, Shanghai, China and M.S. in Electric Power Engineering from North China Electric Power University, Beijing, China.

She worked in Anhui Electric Power Company Ma'anshan Branch, China from 1995 to 1998. She was an engineer in the Power Dispatch Department. She worked on the SCADA system and contributed to several SCADA projects in the distributions and dispatch center.

She presently is a Ph.D. student in Electrical Engineering at The University of Tennessee. Her research interests include non-active power compensation and pricing, and its application in distributed energy systems.

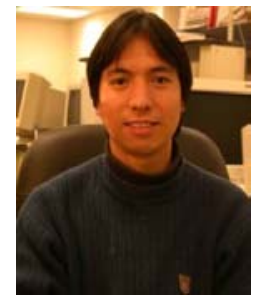

Jianqing Chen (S 2001) received his B.E. in Electrical Engineering from the Harbin Institute of Technology, Harbin, China, and his M.S. in Electrical Engineering from the Zhejiang University, Hangzhou, China.

He worked for Siemens Power Transmission and Distribution for four years before he joined the University of Tennessee.Presently he is a $\mathrm{PhD}$ student in Electrical and Computer Engineering at the University of Tennessee. His research interests include power electronics, motor drives, FACTS, and active power filters. 


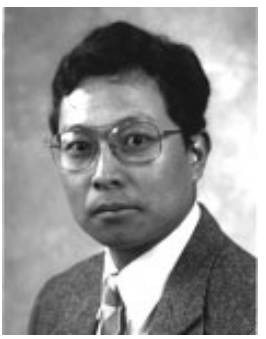

Fang Z. Peng (M 1993 - SM 1996) received the B.S. degree in electrical engineering from Wuhan University of Hydraulic and Electrical Engineering, China, in 1983 and the M.S. and Ph.D. degrees in electrical engineering from Nagaoka University of Technology, Japan, in 1987 and 1990, respectively.

$\mathrm{He}$ joined Toyo Electric Manufacturing Company, Ltd., from 1990 to 1992 as a research scientist and was engaged in research and development of active power filters, flexible ac transmission systems (FACTS) applications and motor drives. From 1992 to 1994, he worked with Tokyo Institute of Technology as a Research Assistant Professor, where he initiated a multilevel inverter program for FACTS applications and a speed-sensorless vector control project. From 1994 to 1997, he was a Research Assistant Professor at University of Tennessee, working for Oak Ridge National Laboratory (ORNL). In 1997, he became a staff member at ORNL, Lead (principal) Scientist of the Power Electronics and Electric Machinery Research Center. He is presently an associate professor in the Department of Electrical and Computer Engineering at Michigan State University.

Dr. Peng has received many awards including the 1996 First Prize Paper Award and the 1995 Second Prize Paper Award of Industrial Power Converter Committee in IEEE/IAS Annual Meeting; the 1996 Advanced Technology Award of the Inventors Clubs of America, Inc., the International Hall of Fame; the 1991 First Prize Paper Award in IEEE Transactions on Industry Applications; and the 1990 Best Paper Award in the Transactions of the IEE of Japan, the Promotion Award of Electrical Academy.

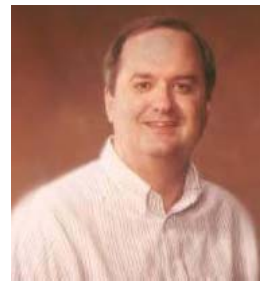

John N. Chiasson (S 1982 - M 1984) received his Bachelor's in Mathematics from the University of Arizona, his M.S. in Electrical Engineering from Washington State University, and his Ph.D. in Controls from the University of Minnesota.

His work in industry started at Boeing Aerospace from 1978 to 1979 in the area of flight controls, guidance and navigation. From 1982-1983, he worked at Control Data in the area of CAD systems, and from 1984-1985 he worked at Honeywell Science and Technology Center in the area of inertial navigation. His latest stint in industry was from 1996-1999 at ABB Daimler-Benz Transportation where he worked in the development of $\mathrm{AC}$ motor propulsion systems, real-time simulators, and the stability analysis of AC propulsion systems. Since 1999, he has been on the faculty of Electrical and Computer Engineering at The University of Tennessee. He does research in the areas of the control of electric motor drives, multilevel converters, hybrid electric vehicles as well as mathematical systems theory.

Dr. Chiasson is an associate editor of the IEEE Transactions on Control Systems Technology. 